\title{
Structure of $\mathrm{As}_{x} \mathrm{Te}_{100-x}(20 \leqslant x \leqslant 60)$ glasses investigated with $x$-ray absorption fine structure, $x$-ray and neutron diffraction, and reverse Monte Carlo simulation
}

\author{
P. Jóvári, ${ }^{1, a)}$ S. N. Yannopoulos, ${ }^{2}$ I. Kaban, ${ }^{3}$ A. Kalampounias, ${ }^{2}$ I. Lishchynskyy, ${ }^{4}$ \\ B. Beuneu, ${ }^{5}$ O. Kostadinova, ${ }^{2}$ E. Welter, ${ }^{6}$ and A. Schöps ${ }^{6}$ \\ ${ }^{1}$ Research Institute for Solid State Physics and Optics, P.O. Box 49, H-1525 Budapest, Hungary \\ ${ }^{2}$ Foundation for Research and Technology Hellas-Institute of Chemical Engineering and High Temperature \\ Chemical Processes (FORTH/ICE-HT), P.O. Box 1414, GR-26504, Rio-Patras, Greece \\ ${ }^{3}$ Institute of Physics, Chemnitz University of Technology, D-09107 Chemnitz, Germany \\ ${ }^{4}$ Vasyl Stefanyk Precarpathian National University, 57 Shevchenko Str., Ivano-Frankivsk, 76025, Ukraine \\ ${ }^{5}$ Laboratoire Léon Brillouin, CEA-Saclay, 91191 Gif sur Yvette Cedex, France \\ ${ }^{6}$ Hamburger Synchrotronstrahlungslabor HASYLAB am Deutschen Elektronen-Synchroton DESY, \\ Notkestrasse 85 D-22603 Hamburg, Germany
}

(Received 16 April 2008; accepted 21 October 2008; published online 4 December 2008)

\begin{abstract}
A systematic and detailed investigation of the structure of $\mathrm{As}_{x} \mathrm{Te}_{100-x}$ glasses $(20 \leqslant x \leqslant 60)$ has been undertaken using a combination of structure-probing techniques including high energy $\mathrm{x}$-ray diffraction, neutron diffraction, and x-ray absorption fine structure measurements at the As and Te $K$ edges. The experimental datasets were modeled simultaneously with the reverse Monte Carlo simulation technique. The results revealed that homonuclear bonding for both As and Te atoms is important over the whole glass concentration region studied. At the stoichiometric composition $\left(\mathrm{As}_{40} \mathrm{Te}_{60}\right)$ the average As-As and $\mathrm{Te}-\mathrm{Te}$ coordination numbers are as high as $1.7 \pm 0.2$ and $1.3 \pm 0.1$, respectively. The number of As-As and Te-Te bonds, as well as the average number of bonds/atom, evolves monotonically with composition. Arsenic atoms are threefold coordinated for all compositions investigated. It has also been shown that, in contrast to the results of previous studies, Te is predominantly twofold coordinated for $x \leqslant 50$. Our results suggest that (i) chemical ordering does not play a decisive role in the formation of short-range order and (ii) similar to some other amorphous tellurides (e.g., $\mathrm{Ge}_{2} \mathrm{Sb}_{2} \mathrm{Te}_{5}, \mathrm{GeSb}_{2} \mathrm{Te}_{4}$, and $\mathrm{As}_{25} \mathrm{Si}_{40} \mathrm{Te}_{35}$ ) binary $\mathrm{As}_{x} \mathrm{Te}_{100-x}(x$ $\leqslant 50$ ) alloys obey the " $8-N$ " rule. A detailed comparison has been advanced between the structural details obtained from the present study and several physicochemical properties of As-Te. The comparison revealed striking similarities between the concentration dependence of structural and physicochemical properties. (C) 2008 American Institute of Physics. [DOI: 10.1063/1.3026591]
\end{abstract}

\section{INTRODUCTION}

At present, amorphous tellurides find a number of applications in phase-change optical data storage, radiometry and optical transmission of information (infrared transmitting optical fibers), and as random access memory devices. ${ }^{1-3}$ As research in these fields is strongly driven by the needs of high-tech industry, physical properties related to the applications are much more systematically investigated than the atomic structure, which is ultimately related to the macroscopic properties itself. This may be the reason why the microscopic origin of fascinating physical phenomena is still not completely understood. A shortcoming of not having yet established microstructure-property relations in noncrystalline tellurides is the lack of a strategic design of new materials for specific applications. As a result, trial-and-error methods are frequently employed to obtain materials' compositions with the desired functionality.

Elucidating the structure of noncrystalline phases is not an easy and straightforward task. Major structure-probing

${ }^{a)}$ Electronic mail: jovari@sunserv.kfki.hu. techniques include $\mathrm{x}$-ray diffraction (XRD) and neutron diffraction (ND) as well as $\mathrm{x}$-ray absorption fine structure (EXAFS). Further, vibrational spectroscopy (Raman and infrared) can also provide an important piece of information on the selected aspects of structural details. ${ }^{4,5}$ The problem becomes more complicated in multicomponent alloys where the description in terms of the partial pair correlation functions (or at least coordination numbers and nearest neighbor distances) is indispensable. The number of these parameters increases steeply with increasing number of components. Already a binary alloy is characterized by three partial pair correlation functions, the separation of which is a tedious task, especially if the nearest neighbor distances of both components are alike as is the case in As-Te glasses.

A challenging aspect of arsenic telluride glasses, which makes their structural studies intriguing, is the fact that these materials are among the very few substances where the densities of the crystalline and glassy phases differ appreciably (of about $13 \%$ for $\mathrm{As}_{40} \mathrm{Te}_{60}$; the differences for $\mathrm{As}_{40} \mathrm{Se}_{60}$ and $\mathrm{As}_{40} \mathrm{~S}_{60}$ are about $4 \%$ and $8 \%$, respectively ${ }^{5,6}$ ) as a result of the different short-range structural order between the two phases. GeTe is another typical example which shows a con- 
trasting local structure in the amorphous and crystalline phases; ${ }^{7}$ a fact that has been considered as an advantage for the enhanced phase-change properties of this material. GeSe is also characterized by large differences between the local order of different phases. In the crystalline state it is characterized by octahedral coordination. As it was revealed by the neutron diffraction (ND) with isotopic substitution study of Petri et al. ${ }^{8}$ upon melting the octahedral structure collapses and the Ge-Se coordination number decreases to $3.2 \pm 0.2$.

Crystalline $\mathrm{As}_{2} \mathrm{Te}_{3}$ was investigated by single crystal XRD. ${ }^{9}$ The structure is monoclinic with two nonequivalent As and three nonequivalent Te sites. Nearest As-Te distances range from 2.68 to $2.93 \AA$. As-As and Te-Te bonds do not exist in crystalline $\mathrm{As}_{2} \mathrm{Te}_{3}$. Due to the presence of nonequivalent sites, the variety of nearest As-As and Te-Te distances is also large (from 3.56 to $4.61 \AA$ for As-As pairs and from 3.70 to $4.43 \AA$ for Te-Te distances).

The structure of amorphous As-Te alloys has been studied in a number of works so far (see Refs. 10-19 and references therein). Evaluating the conclusions of these structural studies, one is faced with contradictory structural models. For example, there is still a confusing picture even for the structure of the stoichiometric $\mathrm{As}_{2} \mathrm{Te}_{3}$ composition. It has been suggested, on one side, that the glass consists of $\mathrm{AsTe}_{3 / 2}$ pyramidal units distributed at random (meaning that the sample is "chemically ordered" but lacks long-range $\operatorname{order}^{16,17}$ and, on the other side, that the amorphous alloy is chemically disordered in view of the presence of homonuclear As-As or Te-Te bonds. ${ }^{10-13}$ The disadvantage of many of the previous works is that a limited number of compositions (sometimes just one alloy) were studied and often only one experimental method was applied. Finally, a third line of reasoning on the structure of $\mathrm{As}_{2} \mathrm{Te}_{3}$ glass invokes the existence of a fraction of As atoms that are octahedrally coordinated in direct analogy with its crystalline counterpart. $^{18,19}$ However, evidence against the existence of octahedral As sites has been presented in Ref. 16.

Obviously, the most detailed study of $\mathrm{As}_{x} \mathrm{Te}_{100-x}$ glasses $(x=20,30,40,50$, and 60) carried out so far is that by $\mathrm{Ma}$ et al., ${ }^{10}$ who applied extended EXAFS at the As and Te $K$ edges and anomalous $\mathrm{X}$-ray scattering. The main conclusions of that study are summarized in the following: (i) As atoms are threefold coordinated over the whole composition range studied; (ii) Te atoms are twofold coordinated in As-rich alloys $(x>40)$, and about $40 \%$ of Te are threefold coordinated in the alloys with $x \leqslant 40$; (iii) the glasses are chemically disordered for all compositions as is evidenced by the existence of homonuclear As-As and Te-Te bonds.

In view of the aforementioned puzzling picture of the structure of As-Te glasses, we have undertaken in the present work a detailed study of the atomic structure of $\mathrm{As}_{x} \mathrm{Te}_{100-x}$ glasses for both the As-rich and Te-rich regimes. In particular, we employ XRD, ND, and EXAFS at the As and Te $K$ edges. As the coherent neutron scattering length of As $(6.58 \mathrm{fm})$ is higher than that of Te $(5.80 \mathrm{fm})$, neutron data are more sensitive to As-As and As-Te correlations than the corresponding x-ray structure factors. As $K$-edge EXAFS is sensitive only to As-As and As-Te correlations, while Te $K$-edge data give information on Te-Te and Te-As dis- tances and coordination numbers. Datasets are analyzed by means of the reverse Monte Carlo (RMC) simulation technique. ${ }^{20}$ This method enables one to obtain partial correlation functions of multicomponent alloys by fitting simultaneously EXAFS datasets with XRD and ND measurements. It has been demonstrated, for example, in the case of amorphous $\mathrm{As}_{25} \mathrm{Si}_{40} \mathrm{Te}_{35},{ }^{21} \mathrm{Ge}_{2} \mathrm{Sb}_{2} \mathrm{Te}_{5},{ }^{22,23}$ and $\mathrm{GeSb}_{2} \mathrm{Te}_{4}$ (Ref. 23) that RMC used in this way is able to distinguish between correlations with similar characteristic distances (such as $\mathrm{Ge}-\mathrm{Sb}$ and $\mathrm{Ge}-\mathrm{Te}$ in the case of $\mathrm{Ge}-\mathrm{Sb}-\mathrm{Te}$ alloys) that cannot be separated by diffraction techniques or their separation would require more subtle techniques (e.g., ND with isotopic substitution). Finally, differential scanning calorimetry (DSC) has been used to determine the glass transition temperature of the glasses.

The rest of the paper is structured as follows. Section II contains the experimental details, i.e., sample preparation and information on EXAFS, diffraction, and DSC experiments. The mathematical formalism and details about the RMC simulation of the data sets obtained are described in Sec. III. The results obtained are presented in Sec. IV where they are discussed in comparison with the previous structural data. Finally, Sec. V summarizes the most important conclusions drawn from the present study.

\section{EXPERIMENTAL}

\section{A. Sample preparation}

The amorphous alloys $\mathrm{As}_{x} \mathrm{Te}_{100-x}$ for $x: 20,34,40,50$, and 60 were prepared from high purity $(99.9999 \%)$ As and Te. Preweighed amounts of As and Te were loaded in thoroughly cleaned and baked at high temperature silica tubes and sealed under vacuum. Due to the moderate glass-forming ability of As-Te mixtures, thin walled and small diameter silica tubes were used, i.e., $4 \mathrm{~mm}$ outer diameter - $3 \mathrm{~mm}$ inside diameter. Sample preparation took place in an inert atmosphere glovebox filled with pure argon in order to avoid oxidation of As metal. Before sealing the tubes the mixture was heated under vacuum at $250{ }^{\circ} \mathrm{C}$ for several hours to remove possible oxide formed at the surface or other volatile substances. At this temperature the vapor pressure of As and Te is negligible. On the other hand, since the materials (in particular As) were stored in the glovebox under inert atmosphere the oxidation of As was almost negligible, while Te has practically negligible reaction with oxygen. Therefore, even if there was an oxide layer at the surface of As chunks, the weight loss after sublimation of this oxide would result practically in no changes in the mole fraction of the alloy. Therefore, the sample composition is reliable to better than $0.1 \%$, i.e., to the error determined by the balance used to weight the elements.

The mixtures were heated slowly to temperatures well above the corresponding liquidus (the phase diagram was taken from Ref. 6) where they were kept for at least $24 \mathrm{~h}$. To ensure homogenization the tubes were shaken frequently. All samples were melted at temperature where the fluidity was enhanced so to ensure homogenization. For all samples, quenching took place from almost the same temperature range, i.e., $800-850^{\circ} \mathrm{C}$. For glasses with $x \leqslant 40$, quenching 
took place to liquid nitrogen to avoid crystallization. The overall conditions of quenching are very similar and we do not expect measurable structural differences due to the slightly different quenching rates. Actually, structural differences in the chalcogenide glasses (mainly in sulfides and less in selenides) can be found only in cases were the various quenching rates differ by almost one order of magnitude ${ }^{4,6}$ which is not the case in our work. Especially in As-Te glasses, the glass transition has been found independent of the conditions of quenching. ${ }^{16}$

\section{B. EXAFS and diffraction measurements}

The XRD experiments on $\mathrm{As}_{20} \mathrm{Te}_{80}, \mathrm{As}_{34} \mathrm{Te}_{66}, \mathrm{As}_{40} \mathrm{Te}_{60}$, $\mathrm{As}_{50} \mathrm{Te}_{50}$, and $\mathrm{As}_{60} \mathrm{Te}_{40}$ were carried out at the BW5 experimental station ${ }^{24}$ at HASYLAB, DESY (Hamburg). The sample material was loaded into thin walled $(0.02 \mathrm{~mm})$ quartz capillaries of $2.0 \mathrm{~mm}$ inner diameter. The size of the incident beam was $1 \times 4 \mathrm{~mm}^{2}$. The energy of the incident beam was $99.8 \mathrm{keV}$. Scattered intensities were recorded by a Ge solid state detector. Raw data were corrected for background, polarization, detector dead time, and variations in detector solid angle. ${ }^{25}$ Due to the high energy of the incident beam, the angle dependence of transmission is not significant over the experimental data range.

The EXAFS measurements on $\mathrm{As}_{20} \mathrm{Te}_{80}, \mathrm{As}_{34} \mathrm{Te}_{66}$, $\mathrm{As}_{40} \mathrm{Te}_{60}, \mathrm{As}_{50} \mathrm{Te}_{50}$, and $\mathrm{As}_{60} \mathrm{Te}_{40}$ were carried out at the beamline Al of HASYLAB. Samples were finely ground, mixed with cellulose, and pressed into pellets. The sample quantity in the pellets was adjusted to the composition of the sample and to the selected edge. The absorption of samples was about $1 / e$ just above the respective absorption edge. Spectra were collected in transmission mode using fixed exit double-crystal $\mathrm{Si}(111)$ and $\mathrm{Si}$ (311) monochromators for As and $\mathrm{Te} K$-edge data, respectively. Incident and transmitted $\mathrm{x}$-ray intensities were measured by ionization chambers filled with gases, the type and pressure of which were adjusted to the corresponding energies. X-ray absorption cross sections $\mu(E)$ were converted to $\chi(k)$ by standard procedures of data reduction using the program VIPER. ${ }^{26}$.

The ND measurements on $\mathrm{As}_{20} \mathrm{Te}_{80}, \mathrm{As}_{40} \mathrm{Te}_{60}, \mathrm{As}_{50} \mathrm{Te}_{50}$, and $\mathrm{As}_{60} \mathrm{Te}_{40}$ were carried out at the $7 \mathrm{C} 2$ diffractometer (LLB, France). Samples were filled into thin walled $(0.1 \mathrm{~mm})$ vanadium containers with $5 \mathrm{~mm}$ diameter. The wavelength of incident radiation was $0.70 \AA$. Raw data were corrected for detector efficiency, empty instrument background, scattering from the sample holder, multiple scattering, and absorption using the standard procedures. Minor inelasticity effects were eliminated by subtracting a parabolic background term by means of the MCGR program. ${ }^{27}$ Experimental XRD and ND structure factors and $k^{3}$ weighted EXAFS $\chi(k)$ curves are shown in Fig. 1.

\section{DSC measurements}

The glass transition temperature $T_{g}$ of amorphous As-Te alloys was determined by conventional DSC (NETZSCH DSC 404). DSC measurements were performed on powdered samples $(\sim 35 \mathrm{mg})$ under argon gas flow. Nonisothermal annealing was carried out from 40 to $250{ }^{\circ} \mathrm{C}$ at the heating rate
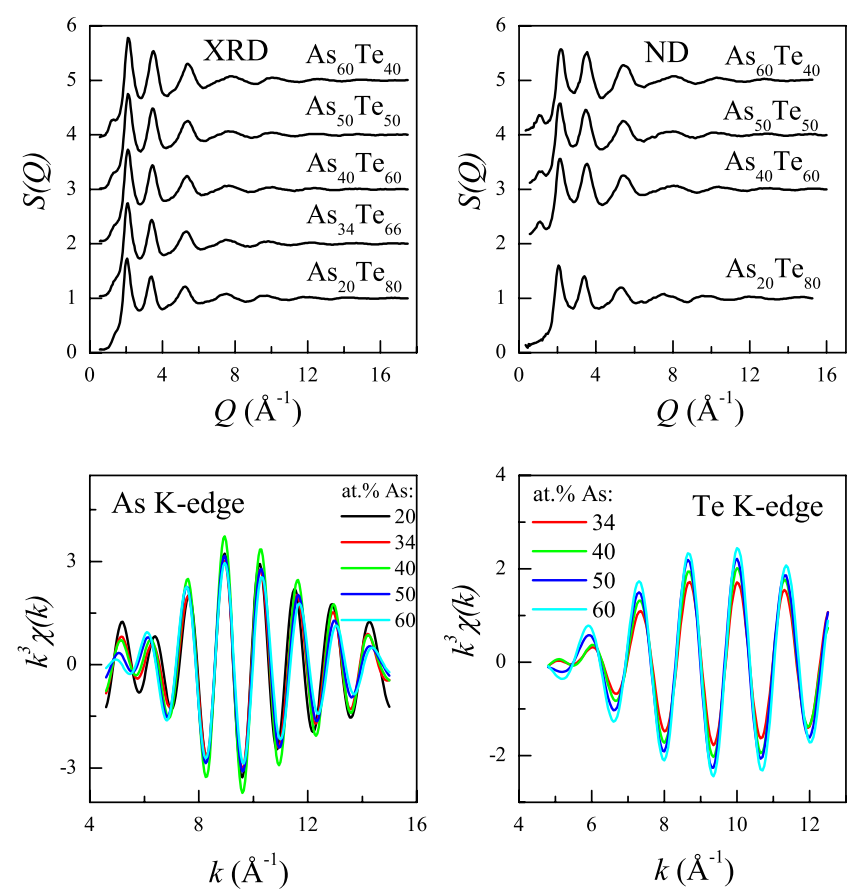

FIG. 1. (Color online) Experimental XRD and ND structure factors, and As and $\mathrm{Te} K$-edge $k^{3} \chi(k)$ curves for $\mathrm{As}_{x} \mathrm{Te}_{100-x}$ glasses $(20 \leqslant x \leqslant 60)$.

of $20 \mathrm{~K} / \mathrm{min}$. Temperature calibration of the calorimeter was performed with high purity elements supplied with the equipment.

\section{RMC modeling}

$\mathrm{XRD}, \mathrm{ND}$, and EXAFS experimental data were modeled simultaneously in the framework of RMC simulation technique. ${ }^{20} \mathrm{RMC}$ is a robust and unsophisticated tool for investigating the structure of disordered materials. For a detailed description of the method we refer to Ref. 28, while recent applications on covalent glasses can be found in Refs. 23 and 29. In order to make this paper self-contained we present here some essential details of the RMC method. In a simulation procedure atoms are placed into a (usually cubic) box and moved around randomly to reproduce (within reasonable limits) the experimental data. In most cases diffraction and EXAFS data are fitted but, in principle, any technique can be modeled if the measured quantity can be calculated from atomic positions. To express model structure factors from atomic coordinates, first the partial pair correlation functions $g_{i j}$ are calculated. In the second step, partial pair correlation functions are transformed to the reciprocal space in the following way:

$$
S_{i j}(Q)-1=\frac{4 \pi \rho}{Q} \int r \sin Q r\left(g_{i j}(r)-1\right) d r,
$$

where $Q$ is the magnitude of the scattering wave vector, $S_{i j}(Q)$ is the corresponding partial structure factor, and $\rho$ is the number density of the sample.

The model total structure factor $\left(S^{\bmod }\right)$ can be obtained by combining the partial structure factors,

$$
S^{\bmod }(Q)=\sum_{i j} w_{i j}(Q) S_{i j}(Q) \text {. }
$$


The weighing coefficients $w_{i j}$ depend on the glass composition and on the type of radiation. For XRD $w_{i j}$ can be calculated from the concentrations $c_{i}$ and atomic form factors $f_{i}$ in the following way:

$$
w_{i j}(Q)=\frac{\left(2-\delta_{i j}\right) c_{i} c_{j} f_{i}(Q) f_{j}(Q)}{\sum_{i j} c_{i} c_{j} f_{i}(Q) f_{j}(Q)} .
$$

In the case of ND the atomic form factors have to be replaced by the coherent neutron scattering lengths. As a result, $w_{i j}$ weighing factors are $Q$-independent for ND.

The $\chi_{i}^{\text {mod }}$ model EXAFS curves can be obtained in a similar way. The main difference is that the EXAFS signal of the $i$ th component contains direct information only on $i-j$ type pair correlations. For example, the model As $K$-edge signal can be expressed from $g_{\mathrm{AsAs}}(r)$ and $g_{\mathrm{AsTe}}(r)$ in the following way:

$$
\begin{aligned}
\chi_{\mathrm{As}}^{\mathrm{mod}}(k)= & 4 \pi \rho\left[c_{\mathrm{As}} \int_{0}^{R} r^{2} \gamma_{\mathrm{AsAs}}(r, k) g_{\mathrm{AsAs}}(r) d r\right. \\
& \left.+c_{\mathrm{Te}} \int_{0}^{R} r^{2} \gamma_{\mathrm{AsTe}}(r, k) g_{\mathrm{AsTe}}(r) d r\right]
\end{aligned}
$$

Here $k$ is the photoelectron wave number, while $\gamma_{\mathrm{AsAs}}$ and $\gamma_{\mathrm{AsTe}}$ are the $r$ - and $k$-dependent photoelectron backscattering coefficients of As and Te atoms. The average number of $j$ type backscatterers in a spherical shell of radius $r$ and thickness $d r$ around an $i$ type absorber atom is $4 \pi \rho c_{j} g_{i j}(r) r^{2} d r$. Thus the above formula simply expresses the $k$-space signal as the superposition of elementary backscattered waves. The backscattering coefficients can be further decomposed in the following way:

$$
\gamma_{i j}(r, k)=A_{i j}(k, r) \sin \left(2 k r+\Phi_{\mathrm{ij}}(k, r)\right) .
$$

Here $A_{i j}(k, r)$ is the amplitude of the backscattering coefficient, while the phase information is given by $\sin (2 \mathrm{kr}$ $\left.+\Phi_{i j}(k, r)\right)$. The $\Phi_{i j}$ phase shift depends on the type of the backscattering atom. A very important consequence of this fact is that a single EXAFS curve may distinguish between different types of neighbors if the difference of their phase shifts is large enough. Using this approach it has been possible to show, for example, that besides $\mathrm{Sb}-\mathrm{Te}$ bonds $\mathrm{Sb}-\mathrm{Ge}$ bonding exists also in amorphous $\mathrm{Ge}_{2} \mathrm{Sb}_{2} \mathrm{Te}_{5}$ (Refs. 22 and 23) and $\mathrm{GeSb}_{2} \mathrm{Te}_{4}{ }^{23}$

$\mathrm{X}$-ray atomic form factors were taken from Ref. 30 . The EXAFS backscattering amplitudes and phases needed to determine $\chi(k)$ from the partial pair distribution functions $g_{i j}(r)$ were calculated using the FEFF8.4 code. $^{31}$ Simulation boxes contained 16000 atoms. The minimum As-As, As-Te, and Te-Te distances were set to $2.3,2.4$, and $2.5 \AA$, respectively. Density values were taken from Ref. 6. At first, unconstrained runs were carried out (without constraining the total number of neighbors of As or Te), while in the second step different coordination constraints were applied to As and Te
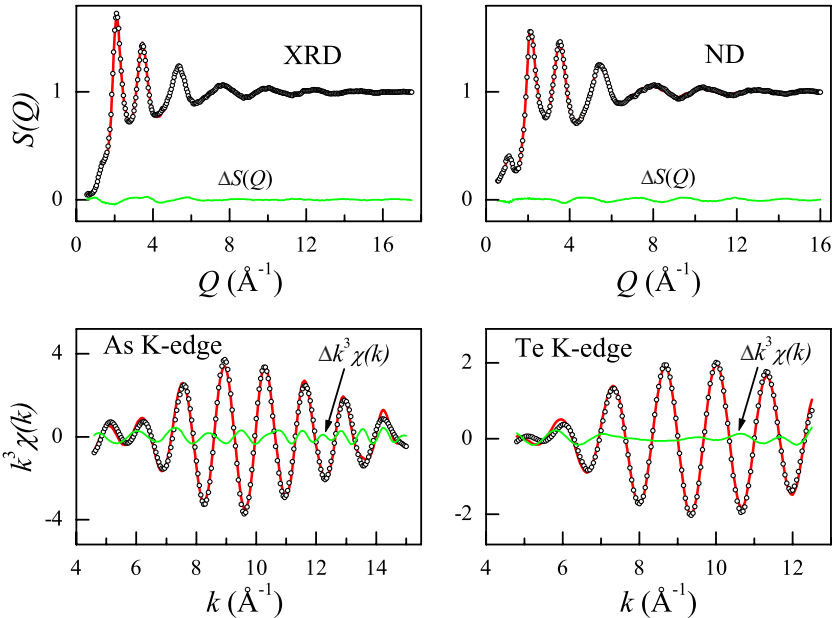

FIG. 2. (Color online) Experimental (circles) and fitted (thick lines) curves of $\mathrm{As}_{40} \mathrm{Te}_{60}$ obtained by the simultaneous RMC modeling of the four measurements (16 000 atoms, constrained run). Thin lines: differences between the experimental and modeled curves.

(see below). As an example, Fig. 2 shows the experimental and RMC simulated $S(Q)$ and $k^{3} \chi(k)$ functions of $\mathrm{As}_{40} \mathrm{Te}_{60}$ obtained by the simultaneous modeling of the four measurements (16 000 atoms, constrained run).

\section{RESULTS AND DISCUSSION}

\section{A. Unconstrained simulations}

Bond distances and coordination numbers obtained by unconstrained simulation runs are listed in Table I. It can be observed that $\left\langle N_{\mathrm{As}}\right\rangle$-the average number of As atom's neighbors-is close to 3 , while $\left\langle N_{\mathrm{Te}}\right\rangle$ - the average number of Te atom's neighbors-is close to 2 over the whole composition range investigated. In the case of As coordination numbers the largest discrepancy $(\sim 9 \%)$ is found for $\mathrm{As}_{20} \mathrm{Te}_{80}$. Taking into account the low As concentration and the fact that the above coordination number was obtained by an unconstrained run, even this deviation is well within the experimental uncertainties.

\section{B. Constrained simulations}

Neither EXAFS nor diffraction data contain information on the coordination number distributions. Even for a one component system these techniques can provide $\langle N\rangle$, the average coordination number, which can be reproduced by several different coordination number distributions. A well

TABLE I. Average number of neighbors of As and Te (denoted by $\left\langle N_{\mathrm{As}}\right\rangle$ and $\left.\left\langle N_{\mathrm{Te}}\right\rangle\right)$ and average coordination numbers $\langle N\rangle$ obtained by the unconstrained RMC simulation. The estimated uncertainty of $\langle N\rangle$ is $5 \%$, while that of $N_{\mathrm{As}}$ and $N_{\mathrm{Te}}$ is about $10 \%$.

\begin{tabular}{lccc}
\hline \hline Composition & $\left\langle N_{\text {As }}\right\rangle$ & $\left\langle N_{\text {Te }}\right\rangle$ & $\langle N\rangle$ \\
\hline $\mathrm{As}_{20} \mathrm{Te}_{80}$ & 2.72 & 2.15 & 2.26 \\
$\mathrm{As}_{34} \mathrm{Te}_{66}$ & 2.94 & 1.99 & 2.31 \\
$\mathrm{As}_{40} \mathrm{Te}_{60}$ & 2.94 & 1.91 & 2.32 \\
$\mathrm{As}_{50} \mathrm{Te}_{50}$ & 2.81 & 2.19 & 2.50 \\
$\mathrm{As}_{60} \mathrm{Te}_{40}$ & 2.99 & 2.26 & 2.70 \\
\hline \hline
\end{tabular}


TABLE II. Coordination numbers and bond lengths $r_{i j}$ for As-Te glasses obtained by constrained RMC simulations. The error of bond lengths is about $0.02 \AA$. The estimated uncertainty of $\langle N\rangle$ is $5 \%$, while that of $N_{\mathrm{AsAs}}, N_{\mathrm{AsTe}}, N_{\mathrm{TeAs}}$, and $N_{\mathrm{TeTe}}$ is about $10 \%-15 \%$. The $N_{i j}$ "partial coordination numbers" can be obtained by integrating $4 \pi \rho c_{j} g_{i j}(r) r^{2}$ up to the first minimum of $g_{i j}(r)$.

\begin{tabular}{lcccccccccc}
\hline \hline Composition & $N_{\text {AsAs }}$ & $N_{\text {AsTe }}$ & $N_{\text {TeAs }}$ & $N_{\text {TeTe }}$ & $\left\langle N_{\text {As }}\right\rangle$ & $\left\langle N_{\text {Te }}\right\rangle$ & $\langle N\rangle$ & $r_{\text {AsAs }}(\AA)$ & $r_{\text {AsTe }}(\AA)$ & $r_{\text {TeTe }}(\AA)$ \\
\hline $\mathrm{As}_{20} \mathrm{Te}_{80}{ }^{a}{ }^{b}$ & 1.05 & 1.92 & 0.48 & 1.62 & 2.97 & 2.10 & 2.27 & 2.50 & 2.56 \\
$\mathrm{As}_{34} \mathrm{Te}_{66}{ }$ & 1.53 & 1.45 & 0.75 & 1.34 & 2.98 & 2.09 & 2.39 & 2.48 & 2.58 \\
$\mathrm{As}_{40} \mathrm{Te}_{60}$ & 1.69 & 1.20 & 0.80 & 1.26 & 2.89 & 2.06 & 2.39 & 2.46 & 2.59 \\
$\mathrm{As}_{50} \mathrm{Te}_{50}$ & 1.84 & 1.03 & 1.03 & 1.03 & 2.87 & 2.06 & 2.47 & 2.46 & 2.59 \\
$\mathrm{As}_{60} \mathrm{Te}_{40}$ & 2.16 & 0.85 & 1.28 & 1.03 & 3.01 & 2.31 & 2.73 & 2.45 & 2.74 \\
\hline \hline
\end{tabular}

${ }^{\mathrm{a}}$ Values obtained without fitting Te $K$-edge data.

${ }^{\mathrm{b}}$ No neutron data were available.

known example is the structure factor of amorphous Si that can be fitted by $100 \%$ and $0 \%$ four-fold coordination as well, while the average coordination number is 4 in both cases. ${ }^{32}$ Thus, the actual shape of the coordination number distribution obtained by an unconstrained simulation run has, in general, no physical meaning. It is only prior physical/chemical knowledge that can help to judge the width of a distribution. We know that $\langle N\rangle=4$ is the result of a sharp distribution in $a$-Si but $\langle N\rangle=12$ is the mean value of a broader distribution in a liquid metal. It is reasonable to assume that in a covalent glass average coordination numbers close to 2 and 3 mean that the majority of Te and As have two and three neighbors, respectively.

Thus, in order to be able to make more definite statements on the structure of As-Te alloys, existing chemical information was built in the simulations in the form of the following coordination constraints: each As was forced to have three neighbors, while Te atoms were allowed to have either two or three neighbors. This structural flexibility was allowed for Te atoms in view of previous studies (e.g., Ref. 10) which concluded that Te can be partly threefold coordinated in the Te-rich region $(x \leqslant 40)$. The type of neighbors was not constrained in these runs. The quality of the fits did not change upon introducing constraints. The results are summarized in Table II.

Since the majority of As atoms (90\%-95\%) satisfied the constraints, only small deviations from the target value $\left(\left\langle N_{\mathrm{As}}\right\rangle=3.0\right)$ were observed. It is remarkable that the average coordination number of Te is very close to 2 for $20 \leqslant x$ $\leqslant 50$. Taking into account that $\mathrm{Te}$ atoms were allowed to have two or three neighbors, this is a strong evidence that $\mathrm{Te}$ is indeed twofold coordinated in $\mathrm{As}_{x} \mathrm{Te}_{100-x}$ glasses over the composition range of $20 \leqslant x \leqslant 50$. The largest deviation was found for $\mathrm{As}_{60} \mathrm{Te}_{40}$ where the coordination number of Te was 2.31. Even this value means that tellurium is mostly $(\sim 70 \%)$ twofold coordinated.

Ma et al. ${ }^{10}$ also suggested that As-As and Te-Te homonuclear bonding play an important role over the whole glassforming region. They found that the number of As-As bonds increases from about 0.2 to 1.5 when As content increases from 20 to 50 at. \%. In parallel, the average number of $\mathrm{Te}-\mathrm{Te}$ bonds per Te atoms decreases from 1.7 to 0.4. Our findings are in qualitative agreement with the above mentioned structural changes; however, there is a clear quantitative difference since our values are significantly higher than those reported in Ref. 10. Deviations are due to two main reasons. The first is the rapid development of electronic structure calculation methods. Ma et al. used the much less accurate backscattering phases and amplitudes of Mac Kale et al. available at that time. ${ }^{33}$ The present day calculation techniques (e.g., the FEFF code $^{31}$ used in our study) provide significantly more accurate parameters. The paper of Ma et $a l$. also strongly relies on anomalous x-ray scattering data. As it is pointed out in Ref. 10 in case of As-Te alloys elastic intensities show only a weak energy dependence which brings about noisy difference structure factors over the whole experimental range and limited useful momentum transfer range (up to about $10 \AA^{-1}$ ) even for the difference structure factors measured around the Te $K$ edge. As a result, the spatial resolution is low making the separation of close $r$-space contributions rather uncertain.

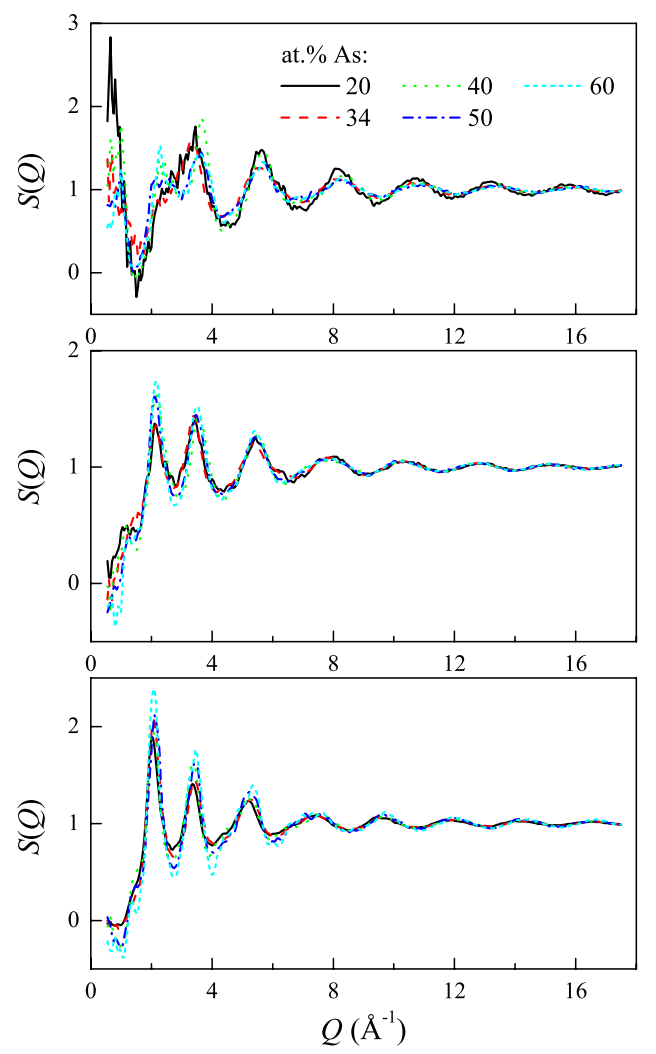

FIG. 3. (Color online) Partial structure factors for As-Te glasses obtained by the constrained simulation runs: Solid line, $\mathrm{As}_{20} \mathrm{Te}_{80}$, dashes; $\mathrm{As}_{34} \mathrm{Te}_{66}$, dot; $\mathrm{As}_{40} \mathrm{Te}_{60}$, dash dots, $\mathrm{As}_{50} \mathrm{Te}_{50}$; and short dashes, $\mathrm{As}_{60} \mathrm{Te}_{40}$. Upper panel: SAsAs(Q); middle panel: $\operatorname{SAsTe}(\mathrm{Q})$; bottom panel: $\mathrm{STeTe}(\mathrm{Q})$. 

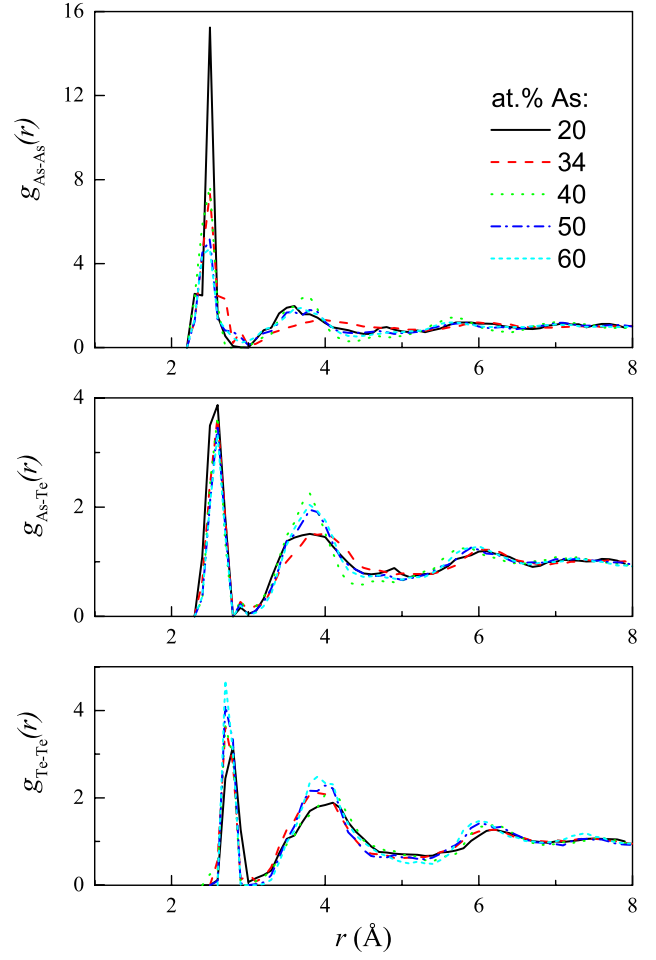

FIG. 4. (Color online) Partial pair correlation functions for As-Te glasses obtained by the constrained simulation runs: solid line, $\mathrm{As}_{20} \mathrm{Te}_{80}$ : dashes, $\mathrm{As}_{34} \mathrm{Te}_{66} ;$ dots, $\mathrm{As}_{40} \mathrm{Te}_{60}$; dash dots, $\mathrm{As}_{50} \mathrm{Te}_{50}$; and short dashes, $\mathrm{As}_{60} \mathrm{Te}_{40}$.

Partial structure factors and pair correlation functions obtained by constrained runs are shown in Figs. 3 and 4. It is evident that the height of the first peak of $g_{\text {AsAs }}(r)$, the As-As partial pair correlation function, changes monotonously with As content. For $x=20$ the first peak is much narrower than for other compositions. For this alloy the contribution of As-As correlations to diffraction measurements is rather low, thus $g_{\text {AsAs }}(r)$ is determined mainly by the As $K$-edge EXAFS measurement. It is well known that EXAFS and diffraction techniques are sensitive to thermal disorder in different ways. Correlated (in-phase) motions of absorber and backscatterer do not contribute to the broadening of bond length distributions. ${ }^{34}$ Therefore, EXAFS peak widths are usually smaller than the values obtained by diffraction techniques. Another consequence of the low As content-and therefore the low weight of $S_{\mathrm{AsAs}}(Q)$ in diffraction measurements-in the case of $\mathrm{As}_{20} \mathrm{Te}_{80}$ is that $g_{\mathrm{AsAs}}(r)$ is somewhat less structured outside the sensitivity range of EXAFS data $(r>3 \AA)$.

In the case of $g_{\mathrm{AsTe}}(r)$ the position and shape of the first peak is practically the same for $34 \leqslant x \leqslant 60$ which means that the length of As-Te bonds is the same for two- and threefold coordinated tellurium atoms. Peak heights and positions of $g_{\mathrm{TeTe}}(r)$ do not show any systematic variation with concentration.

Comparing our results on $\mathrm{As}_{x} \mathrm{Te}_{100-x}$ glasses $(x \leqslant 50)$ with some recent investigations on $\mathrm{GeSb}_{2} \mathrm{Te}_{4}$ and $\mathrm{Ge}_{2} \mathrm{Sb}_{2} \mathrm{Te}_{5}$ (Refs. 22 and 23) and $\mathrm{Si}_{40} \mathrm{As}_{25} \mathrm{Te}_{35}$ (Ref. 21) reveals a common property of these alloys. Although they possess very different glass-forming abilities (As-Te can be vitrified in bulk by melt quenching, while $\mathrm{Ge}-\mathrm{Sb}-\mathrm{Te}$ alloys are identified as marginal glass formers ${ }^{35}$ ) they equally satisfy the 8

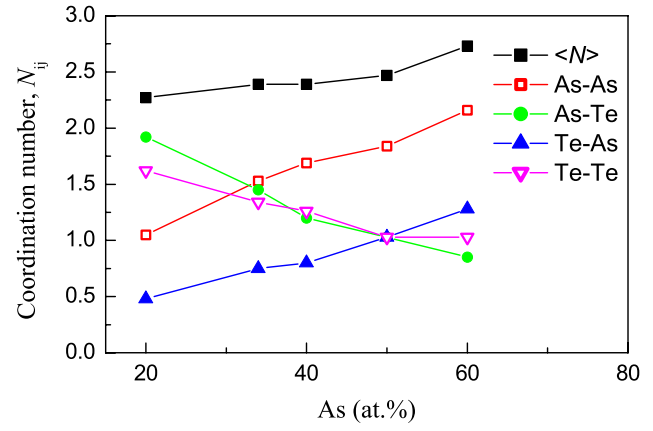

FIG. 5. (Color online) Composition dependences of the coordination numbers for As-Te glasses obtained by constrained RMC simulations.

$-N$ rule. ${ }^{36}$ The above finding is even more interesting if we take into account that $\mathrm{As}_{25} \mathrm{Si}_{40} \mathrm{Te}_{35}$ and the Ge-Sb-Te glasses are characterized by strong heteronuclear bonding preferences (e.g., $\mathrm{Ge}-\mathrm{Te}$ and $\mathrm{Sb}-\mathrm{Te}$ bonds are preferred to $\mathrm{Te}-\mathrm{Te}$ ones in $\mathrm{GeSb}_{2} \mathrm{Te}_{4}$ and $\mathrm{Ge}_{2} \mathrm{Sb}_{2} \mathrm{Te}_{5}$ ), which are not present (or much weaker) in the As-Te alloys, according to the results of the present work. It should be mentioned, however, that the $8-N$ rule is not valid for all glassy tellurides. A recent experimental study revealed that the coordination number of Te is significantly larger than 2 in $\mathrm{Ge}_{15} \mathrm{Te}_{85}{ }^{37}$

\section{Assessment of RMC results}

The average number of As neighbors of As atoms in the "constrained" case of our study is $\sim 1$ for $\mathrm{As}_{20} \mathrm{Te}_{80}$ and $\sim 2$ for $\mathrm{As}_{60} \mathrm{Te}_{40}$. The number of $\mathrm{Te}-\mathrm{Te}$ bonds decreases from $\sim 1.6$ for the glass with 80 at. $\%$ Te down to $\sim 1$ for the glass with 40 at. \% Te. It can also be noticed (see Table II and Fig. 5 ) that the number of homonuclear bonds in the constrained case changes practically monotonically with $x_{\mathrm{As}}$ without showing any extrema at the stoichiometric composition $(x$ $=40)$ as it should be expected if chemical ordering would play an important role in the short-range order of As-Te glasses.

To check whether bonding in these alloys can be considered as "completely random," coordination numbers of the constrained runs are compared with the values obtained by assuming random bonding. Coordination numbers for the completely random case are given by the following simple rules:

(i) Each Te has two neighbors while each As is threefold coordinated.

(ii) There is no preferential bonding in the system. Thus for $\mathrm{As}_{x} \mathrm{Te}_{100-x}$ the average coordination number is 2 $+(x / 100)$ and the probability that a selected bond connects two As atoms is given simply by $(3 x / 2$ $+(x / 100))^{2}$. The same for Te-Te bonding is $(2(100$ $-x) / 2+(x / 100))^{2}$. Then it follows after some straightforward steps that the As-As and Te-Te coordination numbers are equal to $9 x /(200+x)$ and $4(100$ $-x) /(200+x)$, respectively.

Coordination numbers of the completely random model are plotted in Fig. 6. It can be observed that the values determined by RMC are rather close to the curve representing 

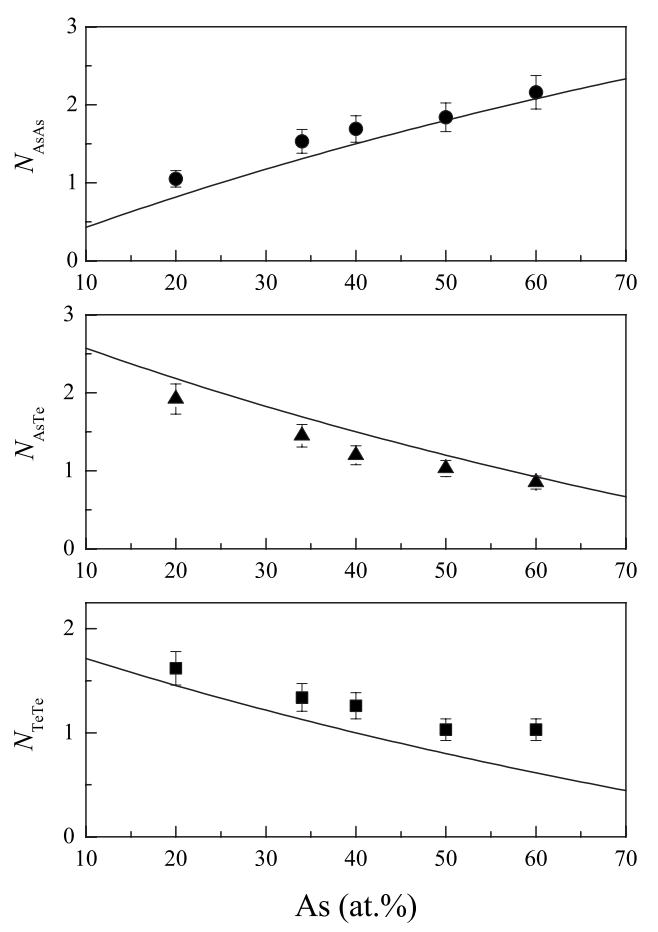

FIG. 6. Comparison of coordination numbers obtained by constrained RMC simulations (symbols) with the completely random bonding model (lines).

the completely random model. The discrepancy is largest for the Te-Te coordination number at $x=60$. This is reasonable because one of the basic assumptions of the model calculations (each Te has two neighbors) does not hold. Given the estimated $10 \%-15 \%$ uncertainty of $N_{\text {AsAs }}, N_{\text {AsTe }}$, and $N_{\mathrm{TeTe}}$ our results suggest that bonding is random over the whole glass-forming region. It was concluded in Ref. 10 that Te is twofold coordinated in the As-rich region $(x>40)$ while the coordination number of Te is 2.4 for $x \leqslant 40$. According to our results the tendency is exactly the opposite: $N_{\mathrm{Te}}$ is very close to 2 for $x \leqslant 50$ while it increases to about 2.3 at $x=60$.

\section{Structural details versus physical properties}

The comparison between structural details and physical properties of glasses is always a useful benchmark in order to assess the validity of the analysis of the experiments and simulations which have provided the structural information in question. In this section we attempt a comparison between the concentration dependence of certain structural parameters estimated by the constrained RMC analysis presented above with the corresponding dependence of various physical properties of the glasses studied.

Glass transition temperature $T_{g}$ is an important parameter reflecting both thermodynamic and rigidity aspects of the glass structure. The As-content dependence of $T_{g}$ determined in this work by DSC is shown in Fig. 7(a). It is important to notice that we have used the same samples in our structural studies and DSC measurements so as to eliminate errors induced by glass preparation details. For comparison, the corresponding dependence of the mean total coordination number, $\langle N\rangle$, is also presented showing a very good coincidence with the $x_{\mathrm{As}}$ dependence of $T_{g}$. This plot reveals the following interesting fact. Both $T_{g}$ and $\langle N\rangle$ exhibit two dif-

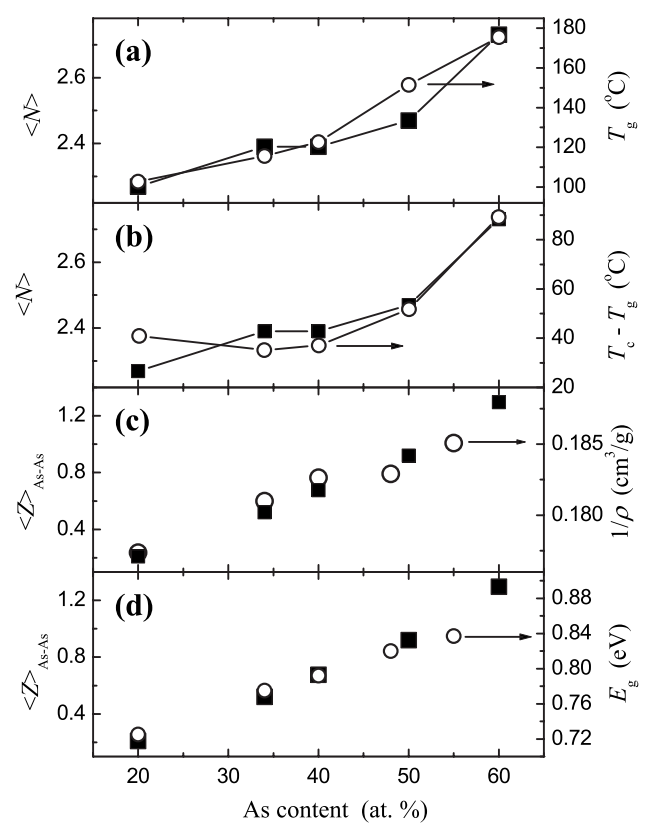

FIG. 7. Composition dependencies of structural and various physiochemical parameters for $\mathrm{As}_{x} \mathrm{Te}_{100-x}$ glasses. (a) $\langle N\rangle$ and $T_{g}$ vs $x_{\mathrm{As}}$. (b) $\langle N\rangle$ and $T_{c}$ $-T_{g}$ vs $x_{\mathrm{As}}$. (c) $\left\langle Z_{\mathrm{AsAs}}\right\rangle$ and reciprocal density vs $x_{\mathrm{As}}$. (d) $\left\langle Z_{\mathrm{AsAs}}\right\rangle$ and $E_{g}$ vs $x_{\mathrm{As}}$. Closed symbols refer to structural data, while open symbols stand for the respective physicochemical parameter.

ferent slopes versus $x_{\mathrm{As}}$ in the Te-rich and As-rich phases. Although there is a qualitative disagreement between these two parameters at $x_{\mathrm{As}}=50$, on quantitative grounds the similarity in slope change is evident. On the contrary, in a previous study ${ }^{10}$ the same comparison was made using the number of homonuclear As-As bonds; in addition, another weak point of that work is that different samples for structural and thermal studies were used. A kink in the slope of the microhardness of As-Te glasses at the stoichiometric composition has also been reported elsewhere ${ }^{16}$ lending support to our findings for the behavior of $T_{g}$ and $\langle N\rangle$ shown in Fig. 7(a). Apart from the glass transition temperature, the stability of the glasses is also reflected in the extent of the temperature interval above $T_{g}$ into which the glass can be heated before crystallization intervenes, i.e., $T_{c}-T_{g}$. The dependence of this parameter versus $x_{\mathrm{As}}$ is shown in Fig. 7(b) together with the dependence of $\langle N\rangle$. Again, a mild dependence of $T_{c}-T_{g}$ in the Te-rich phase is replaced by a stronger one in As-rich glasses. The stability of the glassy state increases with increasing As content as it is seen from Fig. 7(b). This is to be contrasted to the behavior of other As chalcogenides, i.e., As-S and As-Se, where the stoichiometric glass exhibits the highest $T_{g}$ and structural stability where the number of As-As bonds is negligible and therefore cannot be considered as a reason for the improved glass-forming ability as was suggested for As-Te glasses in previous studies. ${ }^{10,16}$ The change from unstable to relatively stable glassy state around 43 at. \% As for As-Te glasses has been reported in Ref. 6. It should be emphasized here that the stability of the glassy state may depend on the energetics of glassy and crystalline phase as well as on the nature of chemical bonds (e.g., strength and cooperativity). The latter is especially important for high As content $(x>50)$ where a significant proportion of 
Te atoms switch from two- to threefold coordination, which may affect the strength of individual chemical bonds. In conclusion, both $T_{g}$ and $T_{c}-T_{g}$ in As-Te glasses exhibit alike concentration dependencies which both follow qualitatively the trend of the mean coordination number. Taking into account that the mean coordination number is a very crude measure of glass stability (differences in As-As, As-Te, and $\mathrm{Te}-\mathrm{Te}$ bond strengths are not taken into account) the above finding is remarkable.

Before turning our attention to nonthermal properties it is interesting to mention that the jump in heat capacity $\Delta C_{p}$ at $T_{p}$, estimated by DSC, decreases linearly with increasing $x_{\mathrm{As}}{ }^{{ }^{\circ}}{ }^{\circ} \Delta C_{p}$ is a measure of the configurational degrees of freedom that become activated as the glass is heated above $T_{g}$. Glasses with network structure, i.e., strong glasses show little change (small jump) in heat capacity at $T_{g}$, while the opposite happens for fragile glasses. Therefore, the decrease in $\Delta C_{p}$ at high As concentrations points to a stronger character of the As-rich glasses. This fact is in accordance with our findings and in particular with the increase in $\langle N\rangle$ and the increase in $\left\langle N_{\mathrm{Te}}\right\rangle$ with $x_{\mathrm{As}}$.

Other parameters whose $x_{\mathrm{As}}$-dependence show striking agreement with our structural data, and in particular with the number of homonuclear As-As bonds, $\left\langle Z_{\mathrm{AsAs}}\right\rangle\left(=x_{\mathrm{As}} N_{\mathrm{AsAs}}\right)$ are the density and the optical energy gap $E_{g}$ Ref. 34 as shown in Figs. 7(c) and 7(d), respectively. We observe that the $x_{\mathrm{As}}$-dependence of both the density and the optical energy gap exhibits a very good agreement with the number of As-As bonds estimated in this work. The optical gap of As-Te glasses shows a linear increase with increasing As content in the glass. This is expected knowing that elemental As has a higher $E_{g}$ value compared to elemental Te. Therefore, the increase in As content in the binary glasses will cause the substitution of Te-Te bonds with As-As bonds and hence the increase in $E_{g}$. As has been suggested elsewhere, ${ }^{4}$ the magnitude of $E_{g}$ correlates with the strength of the chemical bonds building up the glass structure. The presence of the strong As-As bonds at the expense of the weaker Te-Te bonds is responsible for the band gap widening upon increasing $x_{\mathrm{As}}$. The striking similarity between the $x_{\mathrm{As}}$-dependence of $\left\langle Z_{\mathrm{AsAs}}\right\rangle$ and $E_{g}$ indicates the validity of the analysis of the structural data obtained in this work.

To complete the comparison between structural and other physicochemical properties, we illustrate in Fig. 8 the concentration dependence of the number of $\mathrm{Te}-\mathrm{Te}$ bonds, $\left\langle Z_{\mathrm{TeTe}}\right\rangle\left[=\left(1-x_{\mathrm{As}}\right) \times N_{\mathrm{TeTe}}\right]$, together with parameters that depend on the presence of such bonds in As-Te glasses. Data for electronic conductivity measured at $20^{\circ} \mathrm{C}, \sigma_{20}{ }^{\circ} \mathrm{C}$, have been taken from Ref. 6. Both $\left\langle Z_{\mathrm{TeTe}}\right\rangle$ and the logarithm of conductivity exhibit a linear decrease with decreasing number of Te-Te bonds, suggesting that the presence of homonuclear Te-Te bonds is responsible for increased conductivity in Te-rich glasses.

It was also found that the increasing number of $\mathrm{Te}-\mathrm{Te}$ bonds is closely related to the increase in the mean polarizability $\langle\alpha\rangle,{ }^{38}$ as shown in Fig. 8(b). Although a detailed discussion is certainly beyond the scope of the present study the comparison of bond strengths provides a qualitative understanding of the above effect. The fact that $\mathrm{Te}-\mathrm{Te}$ bonds
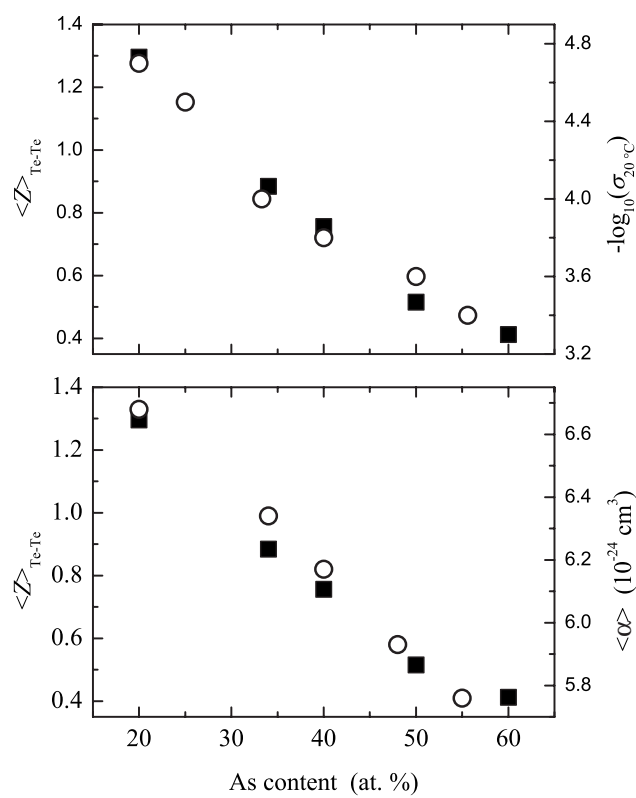

FIG. 8. Composition dependencies of structural and various physiochemical parameters for $\mathrm{As}_{x} \mathrm{Te}_{100-x}$ glasses. (a) $\left\langle Z_{\mathrm{TeTe}}\right\rangle$ and conductivity vs $x_{\mathrm{As}}$. (b) $\left\langle Z_{\mathrm{TeTe}}\right\rangle$ and mean polarizability vs $x_{\mathrm{As}}$. Closed symbols refer to structural data, while open symbols stand for the respective physicochemical parameter.

are much weaker $(38 \mathrm{kcal} / \mathrm{mol})$ than As-Te bonds $(45 \mathrm{kcal} / \mathrm{mol})$ implies that the electron cloud along the $\mathrm{Te}-\mathrm{Te}$ bond is more loosely bound than that of the As-Te bond and hence more polarizable. The dependence of $\langle\alpha\rangle$ on concentration is again linear and bears a very good similarity with $\left\langle Z_{\mathrm{TeTe}}\right\rangle$ as estimated from our structural analysis.

\section{CONCLUSIONS}

$\mathrm{As}_{x} \mathrm{Te}_{100-x}$ glasses $(20 \leqslant x \leqslant 60)$ were studied by high energy XRD, ND, and EXAFS measurements at the As and Te $K$ edges. The RMC simulation technique was used to generate large scale models compatible with experimental data. Analysis of the resulting atomic configurations revealed that homonuclear bonding is important over the whole glassforming region. At the stoichiometric composition $\left(\mathrm{As}_{40} \mathrm{Te}_{60}\right)$ the average As-As and Te-Te coordination numbers amount to $1.7 \pm 0.2$ and $1.3 \pm 0.1$, respectively. As-As bonding is significant in the Te-rich region $(34 \geqslant x)$, while $\mathrm{Te}-\mathrm{Te}$ bonding is considerable in the As-rich region $(x$ $\geqslant 50)$ as well. It has been shown that Te is predominantly twofold coordinated for $x \leqslant 50$, while As is threefold coordinated for all compositions investigated. It can be concluded that chemical ordering does not play an important role in the formation of short-range order of As-Te glasses andsimilar to some other amorphous tellurides-(e.g., $\mathrm{Ge}_{2} \mathrm{Sb}_{2} \mathrm{Te}_{5}, \mathrm{GeSb}_{2} \mathrm{Te}_{4}$, and $\mathrm{As}_{25} \mathrm{Si}_{40} \mathrm{Te}_{35}$ ) glassy As-Te alloys obey the $8-N$ rule for $x \leqslant 50$.

The significance of a structural study lies on its power to provide structure-property relations which are ultimately useful for materials applications. In an effort to step toward this direction we have undertaken extensive comparisons between the structural information obtained in the present study with several physicochemical properties of the As-Te 
glasses. In particular, the total mean coordination number $\langle N\rangle$ was found to correlate qualitatively with thermal parameters, e.g., $T_{g}$ and $T_{c}-T_{g} .\langle N\rangle$ as well as both $T_{g}$ and $T_{c}$ $-T_{g}$ exhibit different slopes below and above the stoichiometric limit. The concentration dependence of the number of As-As bonds exhibits noticeable similarities with the corresponding dependence of the density and the optical energy gap. Finally, the number of Te-Te bonds seems to govern properties such as the conductivity and the mean polarizability of the system.

\section{ACKNOWLEDGMENTS}

I. Kaban acknowledges DESY for the financial support. P. Jóvári was supported by the Bolyai Research Fellowship of the Hungarian Academy of Sciences and by the Hungarian Basic Research Found (OTKA) under Grant No. T048580. The neutron diffraction measurement at Saclay (France) was supported by the European Commission under the 6th Framework Programme through the Key Action: Strengthening the European Research Area, Research Infrastructures under Contract No. HII3-CT-2003-505925. S. N. Yannopoulos and O. Kostadinova acknowledge financial support of the "PENED-03/E $\Delta-887$ " project which is cofunded: $75 \%$ of public financing from the European Union-European Social Fund and $25 \%$ of public financing from the Greek State, Ministry of Development-GSRT; the Hellenic Telecommunications Organization (OTE S.A.) is also thanked for support. I. Lishchynskyy acknowledges DAAD (German Academic Exchange Service) for supporting his research work at TU Chemnitz.

${ }^{1}$ Photoinduced Metastability in Amorphous Semiconductors, edited by A. V. Kolobov, (Wiley-VCH, Weinheim, 2003).

${ }^{2}$ S. Danto, P. Houizot, C. Boussard-Pledel, X.-H. Zhang, F. Smektala, and J. Lucas, Adv. Funct. Mater. 16, 1847 (2006).

${ }^{3}$ M. H. R. Lankhorst, B. W. S. M. M. Ketelaars, and R. A. M. Wolters, Nature Mater. 4, 347 (2005).

${ }^{4}$ S. R. Elliott, Physics of Amorphous Materials, 2nd ed. (Longman Scientific, 1990).

${ }^{5}$ A. Feltz, Amorphous Inorganic Materials and Glasses, (VCH, Weinheim, 1993).

${ }^{6}$ Z. U. Borisova, Glassy Semiconductors (Plenum, New York, 1981).

${ }^{7}$ S. N. Yannopoulos, K. S. Andrikopoulos, G. A. Voyiatzis, A. V. Kolobov, and J. Tominaga, J. Phys.: Condens. Matter 18, 965 (2006).

${ }^{8}$ I. Petri, P. S. Salmon, and H. E. Fischer, J. Phys.: Condens. Matter 11,
7051 (1999)

${ }^{9}$ G. J. Carron, Acta Cryst. 16, 338 (1963).

${ }^{10}$ Q. Ma, D. Raoux, and S. Benazeth, Phys. Rev. B 48, 16332 (1993).

${ }^{11}$ J. C. Wasse, I. Petri, and P. S. Salmon, J. Phys.: Condens. Matter 13, 6165 (2001)

${ }^{12}$ M. Dongol, Th. Gerber, M. Hafiz, M. Abou-Zied, and A. F. Elhady, J. Phys.: Condens. Matter 18, 6213 (2006).

${ }^{13}$ S. Sen, S. Joshi, B. G. Aitken, and S. Khalid, J. Non-Cryst. Solids 354, 4620 (2008).

${ }^{14}$ G. Faigel, L. Gránásy, I. Vincze, and H. de Waard, J. Non-Cryst. Solids 57, 411 (1983).

${ }^{15}$ S. S. K. Titus, S. Asokan, T. S. Panchapagesan, and E. S. R. Gopal, Phys. Rev. B 46, 14493 (1992).

${ }^{16}$ J. Cornet and D. Rossier, J. Non-Cryst. Solids 12, 61 (1973); 12, 85 (1973).

${ }^{17}$ J. R. Fitzpatrick and C. Maghrabi, Phys. Chem. Glasses 12, 105 (1971).

${ }^{18}$ A. Tverjanovich, M. Yagodkina, and V. Strykanov, J. Non-Cryst. Solids 223, 86 (1998).

${ }^{19}$ J. Cornet and D. Rossier, in The Structure of Non Crystalline Materials, edited by Ph. Gaskell (Taylor \& Francis, London, 1977), p. 17.

${ }^{20}$ R. L. McGreevy and L. Pusztai, Mol. Simul. 1, 359 (1988).

${ }^{21}$ I. Kaban, S. Gruner, P. Jóvári, M. Kehr, W. Hoyer, R. G. Delaplane, and M. Popescu, J. Phys.: Condens. Matter 19, 335210 (2007).

${ }^{22}$ P. Jóvári, I. Kaban, J. Steiner, B. Beuneu, A. Schöps, and A. Webb, J. Phys.: Condens. Matter 19, 335212 (2007).

${ }^{23}$ P. Jóvári, I. Kaban, J. Steiner, B. Beuneu, A. Schöps, and A. Webb, Phys. Rev. B 77, 035202 (2008).

${ }^{24}$ R. Bouchard, D. Hupfeld, T. Lippmann, J. Neuefeind, H.-B. Neumann, H. F. Poulsen, U. Rütt, T. Schmidt, J. R. Schneider, J. Süssenbach, and M. von Zimmermann, J. Synchrotron Radiat. 5, 90 (1998).

${ }^{25}$ H. F. Poulsen, H.-B. Neumann, J. R. Schneider, J. Neuefeind, and M. D. Zeidler, J. Non-Cryst. Solids 188, 63 (1995).

${ }^{26}$ K. V. Klementev, J. Phys. D: Appl. Phys. 34, 209 (2001).

${ }^{27}$ L. Pusztai and R. L. McGreevy, Physica B 234-236, 357 (1997).

${ }^{28}$ R. L. McGreevy, J. Phys.: Condens. Matter 13, R8 77 (2001).

${ }^{29}$ O. Gereben, P. Jóvári, L. Temleitner, and L. Pusztai, J. Optoelectron. Adv. Mater. 9, 3021 (2007).

${ }^{30}$ D. Waasmaier and A. Kirfel, Acta Crystallogr., Sect. A: Found. Crystallogr. A51, 416 (1994).

${ }^{31}$ A. L. Ankudinov, B. Ravel, J. J. Rehr, and S. D. Conradson, Phys. Rev. B 58, 7565 (1998)

${ }^{32}$ O. Gereben and L. Pusztai, Phys. Rev. B 50, 14136 (1994).

${ }^{33}$ A. G. Mac Kale, B. W. Vael, A. P. Paulikas, S. K. Chan, and G. S. Knapp, J. Am. Chem. Soc. 110, 3763 (1988).

${ }^{34}$ P. A. Lee, P. H. Citrin, P. Eisenberger, and B. M. Kincaid, Rev. Mod. Phys. 53, 769 (1981).

${ }^{35}$ J. Kalb, F. Spaepen, and M. Wuttig, J. Appl. Phys. 93, 4852 (2003).

${ }^{36}$ N. Mott, Adv. Phys. 16, 49 (1967)

${ }^{37}$ I. Kaban, P. Jóvári, W. Hoyer, and E. Welter, J. Non-Cryst. Solids 353, 2474 (2007).

${ }^{38}$ M. Zavetova, B. Velicky, and V. Vorlicek, Sol. Energy Mater. 8, 33 (1982). 